September 18, 2018

\title{
An explicit solution of the (quantum) elliptic Calogero-Sutherland model ${ }^{1}$
}

\author{
Edwin Langmann \\ Mathematical Physics, Department of Physics, KTH, AlbaNova, SE-106 91 \\ Stockholm, Sweden
}

Dedicated to the memory of Ludwig Pittner

\begin{abstract}
We present explicit formulas for the eigenvalues and eigenfunctions of the elliptic Calogero-Sutherland (eCS) model as formal power series to all orders in the nome of the elliptic functions, for arbitrary values of the (positive) coupling constant and particle number. Our solution gives explicit formulas for an elliptic deformation of the Jack polynomials.
\end{abstract}

\section{Introduction}

The elliptic Calogero-Sutherland (eCS) system is a quantum mechanical model of identical particles moving on a circle and interacting via a two-body potential given by the Weierstrass elliptic function $\wp[\mathrm{C},[\mathrm{Su}, \mathrm{OS}]$. It is defined by the 2 nd order differential operator

$$
H=-\sum_{j=1}^{N} \frac{\partial^{2}}{\partial x_{j}^{2}}+\gamma \sum_{1 \leq j<k \leq N} V\left(x_{j}-x_{k}\right)
$$

where $N=2,3, \ldots$ is the particle number, $-\pi \leq x_{j} \leq \pi$ are coordinates on the circle,

$$
\gamma=2 \lambda(\lambda-1), \quad \lambda>0
$$

\footnotetext{
${ }^{1}$ Contribution to the conference SPT 2004 in Cala Gonone (Sardinia, Italy)
} 
is the coupling constant, and the two-body potential

$$
V(r)=\sum_{m \in \mathbb{Z}} \frac{1}{4 \sin ^{2}[(r+\mathrm{i} \beta m) / 2]}, \quad \beta>0,
$$

which is essentially equal to the Weierstrass elliptic function $\wp$ with periods $2 \pi$ and $\mathrm{i} \beta .^{2}$

The eCS system is known to be integrable in the sense that there exist differential operators of the form

$$
H_{n}=\sum_{j=1}^{N}(-\mathrm{i})^{n} \frac{\partial^{n}}{\partial x_{j}^{n}}+\text { lower order terms }
$$

for all $n=1,2, \ldots, N$, which include the eCS Hamiltonian, $H_{2}=H$, and which all mutually commute, $\left[H_{n}, H_{m}\right]=0$ for $n, m=1,2, \ldots, N$ [S. Moreover, in the trigonometric limit $\beta=\infty$ where the two-body potential reduces to a trigonometric function, the explicit solution of this model was found by Sutherland more than 30 year ago $[\mathrm{Su}]$. In the two-particle case, $N=2$, the eigenvalue equation of the eCS system is equivalent to the Lamé equation studied extensively at the end of the 19th century; see [WW] for a review of the classical results. Recent work on the eCS model include [DI, EK, EFK, FV1, FV2, FGP, S, T].

In this paper we present a generalization of Sutherland's solution to the elliptic case without restrictions on parameters (see the Result in the final section). More specifically, we present explicit formulas for the eigenfunctions $\psi(\mathbf{x} ; \mathbf{n})$ and corresponding eigenvalues $\mathcal{E}(\mathbf{n})$ of the eCS Hamiltonian,

$$
H \psi(\mathbf{x} ; \mathbf{n})=\mathcal{E}(\mathbf{n}) \psi(\mathbf{x} ; \mathbf{n}), \quad \mathbf{x}=\left(x_{1}, \ldots, x_{N}\right),
$$

which are labeled by integer quantum numbers

$$
\mathbf{n}=\left(n_{1}, \ldots, n_{N}\right), \quad n_{1} \geq n_{2} \geq \ldots \geq n_{N},
$$

and which are of the following form,

$$
\psi(\mathbf{x} ; \mathbf{n})=\Phi(\mathbf{x} ; \mathbf{n}) \Psi(\mathbf{x})
$$

where

$$
\Psi(\mathbf{x})=\prod_{1 \leq j<k \leq N} \theta\left(x_{j}-x_{k}\right)^{\lambda}
$$

\footnotetext{
${ }^{2}$ To be precise: $V(z)=\wp(z)+c_{0}$ with $c_{0}=1 / 12-(1 / 2) \sum_{m=1}^{\infty} \sinh ^{-2}[(\beta m) / 2]$.
} 
with

$$
\theta(z)=\sin (z / 2) \prod_{n=1}^{\infty}\left(1-2 q^{2 n} \cos (z)+q^{4 n}\right), \quad q=\mathrm{e}^{-\beta / 2}
$$

essentially the Jacobi Theta function $\vartheta_{1} \cdot{ }^{3}$ The $\Phi$ are symmetric functions of the variables $z_{j}=\mathrm{e}^{\mathrm{i} x_{j}}$, and they are in one-to-one correspondence with the plane waves

$$
S(\mathbf{x} ; \mathbf{n})=\sum_{Q \in S_{N}} \prod_{j=1}^{N} \mathrm{e}^{\mathrm{i} n_{Q j} x_{j}}
$$

which provide a complete set of eigenfunctions in the non-interacting case $\gamma=0$. It is important to note that our solution in the trigonometric limit reduces to Sutherland's: the eigenvalues $\mathcal{E}(\mathbf{n})$ become equal to the well-known expressions

$$
\mathcal{E}_{0}(\mathbf{n})=\sum_{j=1}^{N}\left(n_{j}+\lambda\left[\frac{1}{2}(N+1)-j\right]\right)^{2}
$$

and the functions $\Phi(\mathbf{x} ; \mathbf{n})$ reduce to the Jack polynomials playing a prominent role also in various other contexts in mathematics; see $\mathrm{McD}$, $\mathrm{St}$. We also note that, for $\beta<\infty$, the functions $\Phi(\mathbf{x} ; \mathbf{n})$ are no longer polynomials, and also the eigenvalues $\mathcal{E}(\mathbf{n})$ become much more complicated. In particular, $\Psi$ is not the ground state of the eCS Hamiltonian for $\beta<\infty$. Correspondingly, our solution is by infinite series which, at this point, are only formal: we leave open the important but difficult questions of convergence and resonances (as discussed in more detail below). We only mention the results in Ref. [KT] which suggest that our series solutions have a finite radius of convergence in the nome $q$ of the elliptic functions, and our results suggest that there exists a resummation so that the resonances disappear L4. Moreover, in the trigonometric limit resonances do not appear [L2, and all our infinite series collapse to finite ones.

The present paper is based on our previous results in CL, L1, L2, L3, L4]. Our starting point is a Theorem obtained by quantum field theory techniques in [2] and proven by direct computations in $\mathrm{L} 4$. This theorem suggests to write the eigenfunctions $\Phi(\mathbf{x} ; \mathbf{n})$ as series of particular symmetric functions $\hat{F}(\mathbf{x} ; \mathbf{n})$ which are given by the following explicit formulas,

$$
\hat{F}(\mathbf{x} ; \mathbf{n})=\prod_{j=1}^{N}\left[\oint_{\mathcal{C}_{j}} \frac{\mathrm{d} \xi_{j}}{2 \pi \xi_{j}} \xi_{j}^{n_{j}}\right] \frac{\prod_{1 \leq j<k \leq N} \Theta\left(\xi_{j} / \xi_{k}\right)^{\lambda}}{\prod_{j, k=1}^{N} \Theta\left(\mathrm{e}^{\mathrm{i} x_{j}} / \xi_{k}\right)^{\lambda}}
$$

\footnotetext{
${ }^{3}$ To be precise: $\theta(z)=\vartheta_{1}(z / 2) /\left[2 q^{1 / 4} \prod_{n=1}^{\infty}\left(1-q^{2 n}\right)\right]$.
} 
where

$$
\Theta(\xi)=(1-\xi) \prod_{m=1}^{\infty}\left[\left(1-q^{2 m} \xi\right)\left(1-q^{2 m} / \xi\right)\right],
$$

and the integration contours are nested circles in the complex plane enclosing the unit circle,

$$
\mathcal{C}_{j}: \xi=\mathrm{e}^{\varepsilon j} \mathrm{e}^{\mathrm{i} y_{j}}, \quad-\pi \leq y_{j} \leq \pi, \quad 0<\varepsilon<\beta / N .
$$

Note that the $\hat{F}(\mathbf{x} ; \mathbf{n})$ are symmetric functions of the variables $z_{j}=\mathrm{e}^{\mathrm{i} x_{j}}$, and they can be expanded as Laurent series. A simple but important consequence of the above mentioned theorem is the following.

Corollary: Let

$$
\Phi(\mathbf{x} ; \mathbf{n})=\sum_{\mathbf{m}} \alpha(\underline{\boldsymbol{\mu}} ; \mathbf{n}) \hat{F}(\mathbf{x} ; \mathbf{n}+\underline{\boldsymbol{\mu}})
$$

where the sum is over all

$$
\underline{\boldsymbol{\mu}}=\sum_{1 \leq j<k \leq N} \mu_{j k} \mathbf{E}_{j k} \text { with } \mu_{j k} \in \mathbb{Z} \text { and }\left(\mathbf{E}_{j k}\right)_{\ell}=\delta_{j \ell}-\delta_{k \ell}
$$

for $\ell=1,2, \ldots, N$, and

$$
\alpha(\underline{\boldsymbol{\mu}} ; \underline{\mathbf{0}})=\delta(\underline{\boldsymbol{\mu}}, \underline{\mathbf{0}})+\mathcal{O}(\gamma) .
$$

Then $\psi(\mathbf{x} ; \mathbf{n})$ defined in Eqs. (6) and (7) is an eigenfunction of the eCS Hamiltonian with corresponding eigenvalue $\mathcal{E}(\mathbf{n})$ provided that

$$
\left[\mathcal{E}_{0}(\mathbf{n}+\underline{\boldsymbol{\mu}})-\mathcal{E}(\mathbf{n})\right] \alpha(\underline{\boldsymbol{\mu}} ; \mathbf{n})=\gamma \sum_{j<k} \sum_{\nu \in \mathbb{Z}} S_{\nu} \alpha\left(\underline{\boldsymbol{\mu}}-\nu \mathbf{E}_{j k} ; \mathbf{n}\right)
$$

with $\mathcal{E}_{0}(\mathbf{n})$ in Eq. (10) and

$$
S_{0}=0, \quad S_{\nu}=\frac{\nu}{1-q^{2 \nu}} \quad \text { and } \quad S_{-\nu}=\frac{\nu q^{2 \nu}}{1-q^{2 \nu}} \quad \text { for } \nu>0 .
$$

Note that $S_{\nu}=[1-\delta(\nu, 0)] \nu /\left(1-q^{2 \nu}\right)$ for all integer $\nu$, but we prefer our somewhat less elegant definition which makes manifest that, in the trigonometric limit $q=0, S_{\nu}=0$ for $\nu \leq 0$. This implies that, for $q=0$, Eq. (17) has triangular structure (in a natural sense explained in Ref. [L2]), which implies that the eigenvalue $\mathcal{E}(\mathbf{n})$ is equal to $\mathcal{E}_{0}(\mathbf{n})$. Moreover, one obtains a simple recursion relation to compute all the non-zero $\alpha(\boldsymbol{\mu} ; \mathbf{n})$ in in Eq. (14). It is interesting to note that this solution algorithm for $q=0$ is different 
from Sutherland's, even though is yields the same solution [L2]. We did not realize in L2] that it is possible to obtain an explicit solution of the recursion relations for the coefficients $\alpha(\boldsymbol{\mu} ; \mathbf{n})$ as follows,

$$
\begin{array}{r}
\alpha \underline{\boldsymbol{\mu}} ; \mathbf{n})=\delta(\underline{\boldsymbol{\mu}} ; \underline{\mathbf{0}})+\sum_{s=0}^{\infty} \gamma^{s} \sum_{j_{1}<k_{1}} \cdots \sum_{j_{s}<k_{s}} \sum_{\nu_{1}, \ldots, \nu_{s}=1}^{\infty} \\
\times \frac{\nu_{1} \nu_{2} \cdots \nu_{s} \delta\left(\underline{\boldsymbol{\mu}}, \sum_{r=1}^{s} \nu_{r} \mathbf{E}_{j_{r}, k_{r}}\right)}{\prod_{r=1}^{s} b\left(\sum_{\ell=1}^{r} \nu_{\ell} \mathbf{E}_{j_{\ell}, k_{\ell}} ; \mathbf{n}\right)} \quad \text { for } q=0,
\end{array}
$$

with

$$
b(\underline{\boldsymbol{\mu}} ; \mathbf{n})=\mathcal{E}_{0}(\mathbf{n}+\underline{\boldsymbol{\mu}})-\mathcal{E}_{0}(\mathbf{n}),
$$

where all sums are in fact finite due to the Kronecker delta and certain properties of the functions $\hat{F}(\mathbf{x} ; \mathbf{n})$ discussed in [L2] (Eq. (19) is a simple special case of our general result presented in the last section). This together with the results in Ref. [L2 provides explicit formulas for the Jack polynomials. It is interesting to note that the recursions relations which one gets in Sutherland's algorithm are more complicated and, to our knowledge, have not been solved explicitly for general $N$ (we are only aware of similarly explicit previous results for $N=3$ [PRZ] ). It is also worth noting that, due to translation invariance, the dependence of the eigenfunctions on the center-of-mass coordinate $x_{1}+\cdots+x_{N}$ is trivial, and therefore the functions $\Phi(\mathbf{x} ; \mathbf{n})$ are of the form $\mathrm{e}^{i n_{N}\left(x_{1}+\cdots x_{N}\right)} \Phi(\mathbf{x} ; \tilde{\mathbf{n}})$ where $\tilde{n}_{j}=n_{j}-n_{N}$ obeys $\tilde{n}_{1} \geq \tilde{n}_{2} \geq \ldots \tilde{n}_{N-1} \geq \tilde{n}_{N}=0$. However, it seems that this is not manifest in our explicit formula for these functions, and we therefore seem to get an infinite number of different representations (labeled by $n_{N}$ ) for each distinct Jack polynomial (this is true not only for $q=0$ but also in the elliptic case).

In the rest of the paper we discuss how to explicitly compute the coefficients $\alpha(\boldsymbol{\mu} ; \mathbf{n})$ and eigenvalues $\mathcal{E}(\mathbf{n})$ for the general elliptic case from the Corollary above. The strategy is to find a "good" expansion parameter, allowing to solve Eq. (17) recursively. The obvious parameter is the squared nome $q^{2}$ of the elliptic function [L1]. While this provides a possible solution algorithm, we were able to obtain the explicit solution only up to order $\left(q^{2}\right)^{7}$ for $N=2$ with the help of MAPLE in this way [L4]. The formulas obtained are rather complicated and suggest that it is hopeless to find explicit expressions to all order in $q^{2}$. However, this result motivates a more efficient solution strategy by expanding in the coupling parameter $\gamma$. As we show, the resulting solution algorithm is indeed simpler, and while we obtained the explicit solution up to order $\gamma^{6}$ without the help of MAPLE, it again seems hopeless to obtain explicit formulas to all orders in $\gamma$ in that way. However, this result gives a better understanding of the structure of the solutions, and it led us to a method of solution to all order. The 
key to this was to introduce a parameter $\eta$ "by hand" which efficiently organizes the complexity of the solution, and this allows us to obtain the solution as a power series in $\eta$ to all orders (without the help of MAPLE). From this our explicit formulas for $\mathcal{E}(\mathbf{n})$ and $\alpha(\underline{\boldsymbol{\mu}} ; \mathbf{n})$ of Eq. (17) are obtained by setting $\eta=1$.

In the next three sections we outline the explicit solutions obtained by expanding in $q^{2}, \gamma$ and $\eta$, respectively. For simplicity in notation we restrict this discussion to the simplest non-trivial case $N=2$. The generalization to arbitrary $N$ is straightforward, but we only give the result in the final section. We plan to include a more detailed derivation of this in a future revision of Ref. [L4].

\section{Expanding in $q^{2}$}

For simplicity we restrict ourselves to $N=2$. In this case $\underline{\boldsymbol{\mu}}=\mu \mathbf{E}_{12}$, and (17) simplifies to

$$
\left[\mathcal{E}_{0}\left(\mathbf{n}+\mu \mathbf{E}_{12}\right)-\mathcal{E}(\mathbf{n})\right] \alpha(\mu ; \mathbf{n})=\gamma \sum_{\nu \in \mathbb{Z}} S_{\nu} \alpha(\mu-\nu ; \mathbf{n})
$$

where $\alpha(\mu ; \mathbf{n})$ is short for $\alpha\left(\mu \mathbf{E}_{12} ; \mathbf{n}\right)$. To simplify notation we suppress the dependence on $\mathbf{n}$ in the following. We make the ansatz

$$
\alpha(\mu)=\sum_{\ell=0}^{\infty} \alpha_{\ell}(\mu) q^{2 \ell}, \quad \mathcal{E}=\sum_{\ell=0}^{\infty} \mathcal{E}_{\ell} q^{2 \ell}
$$

and with

$$
\alpha_{\ell}(0)=\delta(\mu, 0) \quad \text { and } \quad \alpha_{\ell}(\mu)=0 \text { for } \mu<-\ell
$$

we obtain by simple computations (expanding the $S_{\nu}$ in geometric series etc.)

$$
\begin{array}{r}
b(\mu) \alpha_{\ell}(\mu)=\sum_{m=1}^{\ell} \mathcal{E}_{m} \alpha_{\ell-m}(\mu)+\gamma \sum_{\nu=1}^{\ell} \nu \alpha_{\ell}(\mu-\nu) \\
+\gamma \sum_{\nu=1}^{\ell} \sum_{m=1}^{\ell / \nu} \nu\left[\alpha_{\ell-\nu m}(\mu-\nu)+\alpha_{\ell-\nu m}(\mu+\nu)\right]
\end{array}
$$

where $b(\mu)=\mathcal{E}_{0}\left(\mathbf{n}+\mu \mathbf{E}_{12}\right)-\mathcal{E}_{0}(\mathbf{n})$. Using $\mathcal{E}_{0}(\mathbf{n})=\left(n_{1}+\lambda / 2\right)^{2}+\left(n_{2}-\lambda / 2\right)^{2}$ we get

$$
b(\mu)=2 \mu(P+\mu), \quad P=n_{1}-n_{2}+\lambda .
$$

It is important to note that Eq. (24) has triangular structure: for each $\ell$ and $\mu \neq 0$ it determines $\alpha_{\ell}(\mu)$ as a finite sum of terms involving only $\alpha_{\ell^{\prime}}\left(\mu^{\prime}\right)$ and $\mathcal{E}_{\ell^{\prime}}$ with $\ell^{\prime}<\ell$ and 
$\alpha_{\ell}\left(\mu^{\prime}\right)$ with $\mu^{\prime}<\mu$, and the equation for $\mu=0$ allows to determine the $\mathcal{E}_{\ell}$ recursively. We also note that the conditions in Eq. (23) is essential for getting a simple recursion procedure. It is straightforward to implement the recursive computation of the $\alpha_{\ell}(\mu)$ and $\mathcal{E}_{\ell}$ in a symbolic computing software like MAPLE or MATHEMATICA. We only give the results for the eigenvalues which we obtained using MAPLE,

$$
\begin{aligned}
& \mathcal{E}_{1}=\frac{1}{P^{2}-1} \gamma^{2} \\
& \mathcal{E}_{2}=\frac{1}{\left(P^{2}-4\right)\left(P^{2}-1\right)}[\left.6\left(P^{2}-2\right) \gamma^{2}-6 \gamma^{3}+\frac{\left(5 P^{2}+7\right)}{4\left(P^{2}-1\right)^{2}} \gamma^{4}\right] \\
& \mathcal{E}_{3}=\frac{1}{\left(P^{2}-9\right)\left(P^{2}-1\right)}[ 12\left(P^{2}-3\right) \gamma^{2}-48 \gamma^{3}+\frac{4\left(15 P^{4}-37 P^{2}-2\right)}{\left(P^{2}-4\right)\left(P^{2}-1\right)^{2}} \gamma^{4} \\
&\left.-\frac{4\left(7 P^{2}+17\right)}{\left(P^{2}-4\right)\left(P^{2}-1\right)^{2}} \gamma^{5}+\frac{\left(9 P^{4}+58 P^{2}+29\right)}{2\left(P^{2}-4\right)\left(P^{2}-1\right)^{4}} \gamma^{6}\right] \\
& \mathcal{E}_{4}=\frac{1}{\left(P^{2}-16\right)\left(P^{2}-1\right)} {\left[4 \frac{\left(7 P^{4}-74 P^{2}+112\right)}{\left(P^{2}-4\right)} \gamma^{2}-180 \gamma^{3}\right.} \\
&+\frac{3\left(365 P^{10}-6662 P^{8}+42249 P^{6}-115640 P^{4}+119816 P^{2}-18528\right)}{2\left(P^{2}-9\right)\left(P^{2}-2\right)^{2}\left(P^{2}-1\right)^{2}} \gamma^{4} \\
&-\frac{3\left(259 P^{8}-3358 P^{6}+11415 P^{4}-4252 P^{2}-25664\right)}{\left(P^{2}-9\right)\left(P^{2}-4\right)^{3}\left(P^{2}-1\right)^{2}} \gamma^{5} \\
&+\frac{2151 P^{10}-18127 P^{8}-10529 P^{6}+293115 P^{4}-501962 P^{2}+79832}{4\left(P^{2}-9\right)\left(P^{2}-4\right)^{3}\left(P^{2}-1\right)^{4}} \gamma^{6} \\
& \\
&+\frac{715 P^{8}-481 P^{6}-43203 P^{4}+94061 P^{2}+104428}{4\left(P^{2}-9\right)\left(P^{2}-4\right)^{3}\left(P^{2}-1\right)^{4}} \gamma^{7} \\
& 64\left(P^{2}-9\right)\left(P^{2}-4\right)^{3}\left(P^{2}-1\right)^{6}
\end{aligned}
$$

We computed $\mathcal{E}_{\ell}$ up to order $\ell=7$ but for obvious reasons do not write down our full result. This result was previously obtained in [FGP up to $\ell=2$ using a different method, and we convinced ourselves that our results agree.

Remark: In the computations discussed above we implicitly assumed that $b(\mu)$ is always different from zero, which is only the case if $\lambda$, and thus $P$, is not an integer. If $b(\mu)$ vanishes we have a resonance, and while it is possible to generalize the algorithm to allow for resonances [L4] we will not discuss this here for simplicity: Throughout this paper we ignore resonances. (As discussed in [L4], we believe that resonances are not a serious problem.) .)

We observe that the $\mathcal{E}_{\ell}$ become more and more complicated with increasing $\ell$, and it seems that they are polynomials in the coupling constant $\gamma$ as follows,

$$
\mathcal{E}_{\ell}=\sum_{s=2}^{2 \ell} \mathcal{E}_{\ell}^{(s)} \gamma^{s}
$$


where $\mathcal{E}_{\ell}^{(s)}$ become more complicated with increasing $s$. It is interesting to note that the formulas for the coefficients $\mathcal{E}_{\ell}^{(s)}$ become somewhat simpler when expanded in partial

fractions. In particular, we found by inspection that all $\mathcal{E}_{\ell}^{(2)}$ we computed can be written in the following simple form,

$$
\mathcal{E}_{\ell}^{(2)}=\frac{\ell}{2} \sum_{k \mid \ell}\left(\frac{1}{(P-k)}-\frac{1}{(P+k)}\right)
$$

(the sum is over all integer divisors $k$ of $\ell$ ). We checked this formula up to $\ell=7$, and by assuming it to be true for all $\ell$ we obtain by a simple computation

$$
\mathcal{E}^{(2)}=\sum_{\ell=0}^{\infty} \mathcal{E}_{\ell}^{(2)} q^{2 \ell}=\sum_{k=1}^{\infty}\left(\frac{1}{P-k}-\frac{1}{P+k}\right) \frac{k q^{2 k}}{2\left(1-q^{2 k}\right)^{2}}
$$

(this conjecture will be proven in the next section). This formula suggests that it is possible to obtain explicit expressions to all orders in $q^{2}$ if one expands in the coupling parameter $\gamma$. We now present an alternative and simpler solution algorithm motivated by this observation.

\section{$3 \quad$ Expanding in $\gamma$}

We now make the ansatz

$$
\alpha(\mu)=\sum_{s=0}^{\infty} \alpha^{(s)} \gamma^{s}, \quad \mathcal{E}=\mathcal{E}_{0}+\sum_{s=1}^{\infty} \mathcal{E}^{(s)} \gamma^{s},
$$

and by simple computations we obtain from Eq. (17)

$$
b(\mu) \alpha^{(s)}(\mu)-\sum_{r=0}^{s} \mathcal{E}^{(s-r)} \alpha^{(r)}(\mu)=\sum_{\nu \in \mathbb{Z}} S_{\nu} \alpha^{(s-1)}(\mu-\nu)
$$

which we can solve with the following ansatz

$$
\alpha^{(0)}(\mu)=\delta(\mu, 0), \quad \alpha^{(s)}(0)=0 \text { for } s=1,2, \ldots
$$

This yields, in particular,

$$
\mathcal{E}^{(s)}=-\sum_{\nu \in \mathbb{Z}} S_{\nu} \alpha^{(s-1)}(-\nu)
$$


The recursion relations in Eqs. (31) are much simpler than the ones in Eq. (24). By inspection we find that the following ansatz is consistent,

$$
\alpha^{(s)}(\mu)=\sum_{\nu_{1}, \ldots, \nu_{s} \in \mathbb{Z}} S_{\nu_{s}} \cdots S_{\nu_{1}} f_{s}\left(\nu_{1}, \ldots, \nu_{s} ; \mu\right)
$$

and by straightforward computations,

$$
\begin{aligned}
f_{1}\left(\nu_{1} ; \mu\right)= & \frac{\delta\left(\mu, \nu_{1}\right)}{b\left(\nu_{1}\right)} \\
f_{2}\left(\nu_{1}, \nu_{2} ; \mu\right)= & \frac{\delta\left(\mu, \nu_{1}+\nu_{2}\right)}{b\left(\nu_{1}+\nu_{2}\right) b\left(\nu_{1}\right)} \\
f_{3}\left(\nu_{1}, \nu_{2}, \nu_{3} ; \mu\right)= & \frac{\delta\left(\mu, \nu_{1}+\nu_{2}+\nu_{3}\right)}{b\left(\nu_{1}+\nu_{2}+\nu_{3}\right) b\left(\nu_{1}+\nu_{2}\right) b\left(\nu_{1}\right)}-\frac{\delta\left(\nu_{2}+\nu_{3}, 0\right) \delta\left(\mu, \nu_{1}\right)}{b\left(\nu_{2}\right) b\left(\nu_{1}\right)^{2}} \\
f_{4}\left(\nu_{1}, \nu_{2}, \nu_{3}, \nu_{4} ; \mu\right)= & \frac{\delta\left(\mu, \nu_{1}+\nu_{2}+\nu_{3}+\nu_{4}\right)}{b\left(\nu_{1}+\nu_{2}+\nu_{3}+\nu_{4}\right) b\left(\nu_{1}+\nu_{2}+\nu_{3}\right) b\left(\nu_{1}+\nu_{2}\right) b\left(\nu_{1}\right)} \\
& -\frac{\delta\left(\nu_{2}+\nu_{3}, 0\right) \delta\left(\mu, \nu_{1}+\nu_{4}\right)}{b\left(\nu_{2}\right) b\left(\nu_{1}\right)^{2} b\left(\nu_{1}+\nu_{4}\right)}-\frac{\delta\left(\nu_{3}+\nu_{4}, 0\right) \delta\left(\mu, \nu_{1}+\nu_{2}\right)}{b\left(\nu_{4}\right) b\left(\nu_{1}+\nu_{2}\right)^{2} b\left(\nu_{1}\right)} \\
& -\frac{\delta\left(\nu_{2}+\nu_{3}+\nu_{4}, 0\right) \delta\left(\mu, \nu_{1}\right)}{b\left(\nu_{3}+\nu_{4}\right) b\left(\nu_{3}\right) b\left(\nu_{1}\right)^{2}}
\end{aligned}
$$

etc. (it is useful to note that the variables $\nu_{j}$ can be permuted in each term). Using Eq. (33) we get

$$
\mathcal{E}^{(s)}=-\sum_{\nu_{1}, \ldots, \nu_{s} \in \mathbb{Z}} S_{\nu_{s}} \cdots S_{\nu_{1}} f_{s-1}\left(\nu_{1}, \ldots, \nu_{s-1} ;-\nu_{s}\right),
$$

in particular, $\mathcal{E}^{(1)}=0$ and

$$
\mathcal{E}^{(2)}=-\sum_{\nu_{2}, \nu_{1}} S_{\nu_{2}} S_{\nu_{1}} \frac{\delta\left(\nu_{1}+\nu_{2}, 0\right)}{b\left(\nu_{1}\right)}=-\sum_{\nu=1}^{\infty} S_{\nu} S_{-\nu}\left(\frac{1}{b(\nu)}+\frac{1}{b(-\nu)}\right)
$$

which proves Eq. (29). With the formulas given above one can easily write down similarly explicit formulas for $\mathcal{E}^{(s)}$ for $s=2,3,4,5$.

Computing the functions $f_{s}$ up to $s=6$ we observed the following simple patterns: it seems that the building blocks for the solution are the following expressions,

$$
G_{\ell}^{(s)}=\sum_{\nu_{1}, \ldots, \nu_{s} \in \mathbb{Z}} S_{\nu_{s}} \cdots S_{\nu_{1}} \sum_{k_{1}, \ldots, k_{s-1}=0}^{\infty} \delta\left(k_{1}+\cdots+k_{s-1}-\ell\right) \frac{\delta\left(\sum_{\ell=1}^{s} \nu_{s}, 0\right)}{\prod_{r=1}^{s-1} b\left(\sum_{\ell=1}^{r} \nu_{\ell}\right)^{1+k_{r}}}
$$


With that we can write the coefficients of the eigenvalues in a simple manner as follows,

$$
\begin{aligned}
\mathcal{E}^{(2)}= & -G_{0}^{(2)} \\
\mathcal{E}^{(3)}= & -G_{0}^{(3)} \\
\mathcal{E}^{(4)}= & -G_{0}^{(4)}+G_{0}^{(2)} G_{1}^{(2)} \\
\mathcal{E}^{(5)}= & -G_{0}^{(5)}+G_{0}^{(3)} G_{1}^{(2)}+G_{0}^{(2)} G_{1}^{(3)} \\
\mathcal{E}^{(6)}= & -G_{0}^{(6)}+G_{0}^{(4)} G_{1}^{(2)}+G_{0}^{(2)} G_{1}^{(4)} \\
& +G_{0}^{(3)} G_{1}^{(3)}-G_{0}^{(2)}\left[G_{1}^{(2)}\right]^{2}-\left[G_{0}^{(2)}\right]^{2} G_{2}^{(2)}
\end{aligned}
$$

This suggests that

$$
\mathcal{E}^{(s)}=\sum_{r=1}^{\infty}(-1)^{r}(\cdots) \delta\left(s_{1}+\cdots s_{r}, s\right) \delta\left(k_{1}+\cdots+k_{r}, r-1\right) G_{k_{1}}^{\left(s_{1}\right)} \cdots G_{k_{r}}^{\left(s_{r}\right)}
$$

where ' $(\cdots)$ ' are possible combinatorial factors which might appear at higher order but, up to $s=6$, all are equal to 1 .

We find that, for $s>6$, nontrivial combinatorial factors in the formula above appear, and this destroys the hope that we can find a closed formula for $\mathcal{E}^{(s)}$ for arbitrary $s$ in this way. Still, Eq. (40) suggest that we can write $\mathcal{E}$ in a simple manner using the following quantities,

$$
G_{k}=\sum_{s=2}^{\infty} G_{k}^{(s)} \gamma^{s}
$$

and the formulas above suggest,

$$
\mathcal{E}=\mathcal{E}_{0}-G_{0}+G_{0} G_{1}-G_{0}\left[G_{1}\right]^{2}-\left[G_{0}\right]^{2} G_{2}+\ldots
$$

where the dots are higher order terms. We will prove and extend this formula in the next section: We will obtain an explicit formula of the following kind,

$$
\mathcal{E}=\mathcal{E}_{0}+\sum_{n=1} \tilde{\mathcal{E}}_{n}, \quad \tilde{\mathcal{E}}_{n}=\sum_{r_{1}, \ldots, r_{n}}(\cdots) G_{r_{1}} \cdots G_{r_{n}}
$$

with certain combinatorial factor ' $(\cdots)$ ' which we will compute explicitly. It is interesting to note that $\tilde{\mathcal{E}}_{n}$ is of order $q^{2 n}$ (since all $G_{s}$ are of order $q^{2}$ ), and thus this formula allows to deduce in a simple manner the series expansion in $q^{2}$. However, the term $\tilde{\mathcal{E}}_{n}$ contributes to all the $\left(q^{2}\right)^{m}$-terms $m \geq n$, which explains why our expansion in $q^{2}$ yielded so complicated expressions. We stress that, since $\mathcal{E}_{n}=\mathcal{O}\left(q^{2 n}\right)$, Eq. (50) still is an expansion the $q^{2}$ and has, as we believe, a finite radius of convergence. 


\section{Expanding in $\eta$}

The results in the previous section led us to a more powerful solution strategy which we now explain. Defining an operator $\mathbb{S}$ as follows,

$$
\mathbb{S} f(\mu):=\sum_{\nu \in \mathbb{Z}} S_{\nu} f(\mu-\nu)
$$

we can write Eq. (17) as

$$
[b(\mu)-\tilde{\mathcal{E}}] \alpha(\mu)=\gamma \mathbb{S} \alpha(\mu)
$$

where

$$
\tilde{\mathcal{E}}=\mathcal{E}-\mathcal{E}_{0}
$$

Making the ansatz

$$
\alpha(\mu)=\delta(\mu, 0)+\sum_{s=1}^{\infty} \tilde{\alpha}_{s}(\mu) \gamma^{s}
$$

we get $[b(\mu)-\tilde{\mathcal{E}}] \tilde{\alpha}_{s}(\mu)=\mathbb{S} \tilde{\alpha}_{s-1}(\mu)$, and thus

$$
\begin{array}{r}
\alpha(\mu)=\sum_{s=0}^{\infty} \gamma^{s}\left([b(\mu)-\tilde{\mathcal{E}}]^{-1} \mathbb{S}\right)^{s} \delta(\mu, 0)= \\
\sum_{s=0}^{\infty} \gamma^{s} \sum_{\nu_{1}, \ldots, \nu_{s} \in \mathbb{Z}} S_{\nu_{s}} \cdots S_{\nu_{1}} \frac{1}{[b(\mu)-\tilde{\mathcal{E}}]} \frac{1}{\left[b\left(\mu-\nu_{s}\right)-\tilde{\mathcal{E}}\right]} \frac{1}{\left[b\left(\mu-\nu_{s}-\nu_{s-1}\right)-\tilde{\mathcal{E}}\right]} \\
\cdots \frac{1}{\left[b\left(\mu-\nu_{s}-\cdots-\nu_{2}\right)-\tilde{\mathcal{E}}\right]} \delta\left(\mu-\nu_{s}-\nu_{s-1}-\cdots-\nu_{1}, 0\right)= \\
\sum_{s=0}^{\infty} \gamma^{s} \sum_{\nu_{1}, \ldots, \nu_{s} \in \mathbb{Z}} S_{\nu_{s}} \cdots S_{\nu_{1}} \frac{\delta\left(\mu, \sum_{r=1}^{s} \nu_{r}\right)}{\prod_{r=1}^{s}\left[b\left(\mu-\sum_{\ell=r+1}^{s} \nu_{\ell}\right)-\tilde{\mathcal{E}}\right]} .
\end{array}
$$

Setting $\mu=0$ in Eq. (45) gives $\tilde{\mathcal{E}}=-\gamma \mathbb{S} \alpha(0)$, which implies

$$
\tilde{\mathcal{E}}=-\sum_{s=0}^{\infty} \gamma^{s+1} \sum_{\nu_{1}, \ldots, \nu_{s+1} \in \mathbb{Z}} S_{\nu_{s+1}} \cdots S_{\nu_{1}} \frac{\delta\left(\sum_{r=1}^{s+1} \nu_{r}, 0\right)}{\prod_{r=1}^{s}\left[b\left(\mu-\sum_{\ell=r+1}^{s+1} \nu_{\ell}\right)-\tilde{\mathcal{E}}\right]}
$$

It is easy to see that the $s=0$ term here vanishes, and by a shifting the summation variable we obtain the following equation determining $\tilde{\mathcal{E}}$,

$$
\tilde{\mathcal{E}}=-G(\tilde{\mathcal{E}})
$$


where

$$
G(\xi):=\sum_{s=2}^{\infty} \gamma^{s} \sum_{\nu_{1}, \ldots, \nu_{s} \in \mathbb{Z}} S_{\nu_{s}} \cdots S_{\nu_{1}} \frac{\delta\left(\sum_{r=1}^{s} \nu_{r}, 0\right)}{\prod_{r=1}^{s-1}\left[b\left(\sum_{\ell=1}^{r} \nu_{\ell}\right)-\xi\right]} .
$$

We now observe that the functions $G(\xi)$ has a Taylor expansion as follows,

$$
G(\xi)=\sum_{k=0}^{\infty} G_{k} \xi^{k}
$$

with

$$
\begin{gathered}
G_{k}=\left.\frac{1}{k !} \frac{\mathrm{d}^{k}}{\mathrm{~d} \xi^{k}} \sum_{s=2}^{\infty} \gamma^{s} \sum_{\nu_{1}, \ldots, \nu_{s} \in \mathbb{Z}} S_{\nu_{s}} \cdots S_{\nu_{1}} \frac{\delta\left(\sum_{r=1}^{s} \nu_{r}, 0\right)}{\prod_{r=1}^{s-1}\left[b\left(\sum_{\ell=1}^{r} \nu_{\ell}\right)-\xi\right]}\right|_{\xi=0} \\
=\sum_{s=1}^{\infty} \gamma^{s} \sum_{\nu_{1}, \ldots, \nu_{s} \in \mathbb{Z}} \prod_{r=1}^{s} S_{\nu_{r}} \sum_{k_{1}, \ldots, k_{s}=0}^{\infty} \delta\left(k, \sum_{r=1}^{s} k_{r}\right) \frac{\delta\left(\sum_{r=1}^{s} \nu_{r}, 0\right)}{\prod_{r=1}^{s-1}\left[b\left(\sum_{\ell=1}^{r} \nu_{\ell}\right)-\xi\right]^{1+k_{s}}} .
\end{gathered}
$$

Note that these are exactly the quantities which we found empirically in the last Section. To solve Eq. (50) efficiently we replace it by

$$
\tilde{\mathcal{E}}=-\eta \sum_{k=0}^{\infty} G_{k}(\tilde{\mathcal{E}})^{k}
$$

where we introduce a parameter $\eta$ serving as useful book keeping device (we set $\eta=1$ at the end of the computation). It is straightforward to compute the Taylor series of $\tilde{\mathcal{E}}$ recursively: With the Ansatz

$$
\tilde{\mathcal{E}}=\sum_{k=1}^{\infty} \tilde{\mathcal{E}}_{k} \eta^{k}
$$

we get by simple computations

$$
\begin{aligned}
\tilde{\mathcal{E}}_{1} & =-G_{0} \\
\tilde{\mathcal{E}}_{2} & =G_{0} G_{1} \\
\tilde{\mathcal{E}}_{3} & =-\left[G_{0}\right]^{2} G_{2}-G_{0}\left[G_{1}\right]^{2} \\
\tilde{\mathcal{E}}_{4} & =3\left[G_{0}\right]^{2} G_{1} G_{2}+G_{0}\left[G_{1}\right]^{3}+\left[G_{0}\right]^{3} G_{3} \\
\tilde{\mathcal{E}}_{5} & =-4\left[G_{0}\right]^{3} G_{1} G_{3}-6\left[G_{0}\right]^{2}\left[G_{1}\right]^{2} G_{2}-2\left[G_{0}\right]^{3}\left[G_{2}\right]^{2}-\left[G_{0}\right]^{3} G_{4}-G_{0}\left[G_{1}\right]^{4}
\end{aligned}
$$

etc. This suggests that

$$
\tilde{\mathcal{E}}_{n}=(-1)^{n} \sum_{k_{0}, k_{1}, \cdots, k_{n-1}=0}^{\infty} \delta\left(n-1, \sum_{j=1}^{n-1} j k_{j}\right) \delta\left(n, \sum_{j=0}^{n-1} k_{j}\right)\left(\begin{array}{c}
n-1 \\
k_{0}, \ldots, k_{n-1}
\end{array}\right) \prod_{j=0}^{n-1}\left[G_{j}\right]^{k_{j}}
$$


for all $n=1,2, \ldots$ (we checked that using MAPLE up to $n=15$ ). This result is, in fact, a classical theorem due to Lagrange: ${ }^{4}$ the equation determining $\tilde{\mathcal{E}}$ is of the form $\tilde{\mathcal{E}}=-\eta G(\tilde{\mathcal{E}})$, and thus Lagrange's theorem as stated in WW, Paragraph 7.32, implies

$$
\tilde{\mathcal{E}}=\left.\sum_{n=1}^{\infty} \frac{(-\eta)^{n}}{n !} \frac{\mathrm{d}^{n-1}}{\mathrm{~d} y^{n-1}} G(y)^{n}\right|_{y=0}
$$

equivalent to Eq. (57).

\section{Conclusions}

It is possible to generalize the results of the previous two sections to arbitrary particle

numbers $N$. We intend to give the details in a future revision of Ref. [L4] and quote here only the result.

Result: The eigenvalues of the elliptic eCS model are given by

$$
\begin{array}{r}
\mathcal{E}(\mathbf{n})=\mathcal{E}_{0}(\mathbf{n})+\sum_{n=1}^{\infty}(-1)^{n} \sum_{k_{0}, \cdots, k_{n-1}=0}^{\infty} \\
\times \delta\left(n-1, \sum_{j=1}^{n-1} j k_{j}\right) \delta\left(n, \sum_{j=1}^{n-1} k_{j}\right)\left(\begin{array}{c}
n-1 \\
k_{0}, \ldots, k_{n-1}
\end{array}\right) \prod_{j=0}^{n-1}\left[G_{j}(\mathbf{n})\right]^{k_{j}}
\end{array}
$$

with $\mathcal{E}_{0}(\mathbf{n})=\sum_{j=1}^{N}\left(n_{j}+\lambda\left[\frac{1}{2}(N+1)-j\right]\right)^{2}, n_{1} \geq n_{2} \geq \ldots n_{N}$ integers, and

$$
\begin{aligned}
G_{k}(\mathbf{n})=\sum_{s=2}^{\infty} & \gamma^{s} \sum_{j_{1}<k_{1}} \cdots \sum_{j_{s}<k_{s}} \sum_{\nu_{1}, \ldots, \nu_{s} \in \mathbb{Z}} S_{\nu_{s}} \cdots S_{\nu_{1}} \sum_{\ell_{1}, \ldots, \ell_{s}=0}^{\infty} \\
& \times \delta\left(k, \sum_{r=1}^{s} \ell_{r}\right) \frac{\delta\left(\sum_{r=1}^{s} \nu_{r} \mathbf{E}_{j_{r} k_{r}}, \underline{\mathbf{0}}\right)}{\prod_{r=1}^{s-1} b\left(\sum_{\ell=1}^{r} \nu_{\ell} \mathbf{E}_{j_{\ell} k_{\ell}} ; \mathbf{n}\right)^{1+\ell_{s}}}
\end{aligned}
$$

where $b(\underline{\boldsymbol{\mu}} ; \mathbf{n})=\mathcal{E}_{0}(\mathbf{n}+\underline{\boldsymbol{\mu}})-\mathcal{E}_{0}(\mathbf{n}), S_{\nu}=[1-\delta(\nu, 0)] \nu /\left(1-q^{2 \nu}\right)$ and $\mathbf{E}_{j k}$ in Eq. (15). The corresponding eigenfunctions are given by Eqs. (6), (7) and (14)-(15) with the coefficients

$$
\alpha(\underline{\boldsymbol{\mu}})=\delta(\underline{\boldsymbol{\mu}}, \underline{\mathbf{0}})+\sum_{s=1}^{\infty} \gamma^{s} \sum_{j_{1}<k_{1}} \cdots \sum_{j_{s}<k_{s}} \sum_{\nu_{1}, \ldots, \nu_{s} \in \mathbb{Z}} S_{\nu_{s}} \cdots S_{\nu_{1}}
$$

\footnotetext{
${ }^{4}$ I thank S.G. Rajeev and G. Lindblad for helpful discussions on this.
} 


$$
\times \frac{\delta\left(\underline{\boldsymbol{\mu}}, \sum_{r=1}^{s} \nu_{r} \mathbf{E}_{j_{r} k_{r}}\right)}{\prod_{r=1}^{s}\left[b\left(\sum_{\ell=1}^{r} \nu_{\ell} ; \mathbf{n}\right)-\mathcal{E}(\mathbf{n})+\mathcal{E}_{0}(\mathbf{n})\right]} .
$$

There is an even more explicit formula for the coefficients $\alpha(\underline{\boldsymbol{\mu}})$ which we plan to give elsewhere.

We do not label this as theorem since we did not check the details of our formulas here carefully enough to be sure that there are no typos and/or (minor) mistakes. ${ }^{5}$ The purpose of this paper was to make available the explicit solution of the eCS model which we announced in two recent meetings, and to describe the last part of a somewhat lengthy journey leading us to this result. We feel that we are not at the end of this journey yet: quite some work remains to be done to understand this result.

Anyway, it seems fair to say that the eCS model is an exactly solved model now.

\section{Acknowledgments}

I would like to thank V.B. Kuznetsov for helpful discussions and Martin Hallnäs for reading the manuscript. This work was supported by the Swedish Science Research Council (VR) and the Göran Gustafsson Foundation.

\section{References}

[C] Calogero F.: Solution of the one-dimensional N body problems with quadratic and/or inversely quadratic pair potentials, J. Math. Phys. 12, 419 (1971)

[CL] Carey A.L. and Langmann E.: Loop groups, anyons and the Calogero-Sutherland model. Commun. Math. Phys. 2011 (1999)

[DI] Dittrich J. and Inozemtsev V.I.: On the structure of eigenvectors of the multidimensional Lamé operator, J. Phys. A: Math. Gen. 26 (1993) L753

[EK] Etingof P.I. and Kirillov A.A.: Representation of affine Lie algebras, parabolic differential equations and Lamé functions. Duke Math. J. 74, 585 (1994)

\footnotetext{
${ }^{5}$ We finished this paper under the pressure of a deadline. Moreover, we are aware of our unfortunate tendency to make errors when copying formulas from notes to a LaTex file.
} 
[EFK] Etingof P. I., Frenkel I. B., and Kirillov A. A.: Spherical functions on affine Lie groups, Duke Math. J. 80, 59 (1995) arXiv:hep-th/9407047

[FGP] Fernández Núñez J., García Fuertes W., and Perelomov A.M.: A perturbative approach to the quantum elliptic Calogero-Sutherland model, Phys. Lett. A 307, 233 (2003)

[FV1] Felder G. and Varchenko A.: Integral representation of solutions of the elliptic Knizhnik-Zamolodchikov-Bernard equations. Int. Math. Res. Notices No. 5, 221 (1995)

[FV2] Felder G. and Varchenko A.: Three formulas for eigenfunctions of integrable Schroedinger operators. hep-th/9511120

[KT] Komori Y., and Takemura K.: The perturbation of the quantum Calogero-MoserSutherland system and related results, Commun. Math. Phys. 227, 93 (2002) math.qa/0009244

[L1] Langmann E.: Anyons and the elliptic Calogero-Sutherland model, Lett. Math. Phys. 54, 279 (2000) math-ph/0007036

[L2] Langmann E.: Second quantization of the elliptic Calogero-Sutherland model, Comm. Math. Phys. 247, 321 (2004) math-ph/0102005

[L3] Langmann E.: Algorithms to solve the (quantum) Sutherland model, J. Math. Phys. 42, 4148 (2001) math-ph/0104039

[L4] Langmann E.: A perturbative algorithm to solve the (quantum) elliptic CalogeroSutherland model, math-ph/0401029

[McD] Macdonald I.G.: Symmetric functions and Hall polynomials. Oxford Mathematical Monographs. Oxford: Clarendon Press 1979

[OS] Olshanetsky M.A. and Perelomov A.M.: Quantum completely integrable systems connected with semisimple Lie algebras, Lett. Math. Phys. 2, 7 (1977)

[PRZ] Perelomov A. M., Ragoucy E. and Zaugg P.: Explicit solution of the quantum three-body Calogero-Sutherland model, J. Phys. A 31, L559 (1998) hep-th/9805149

[S] Sklyanin E.K.: Separation of variables. New trends. Prog. Theor. Phys. Suppl. No. 118,35 (1995) 
[St] Stanley R.P.: Some properties of Jack symmetric functions. Adv. in Math. 77, 76 (1989)

[Su] Sutherland B.: Exact results for a quantum many body problem in one-dimension. II. Phys. Rev. A5 1372 (1972)

[T] Takemura K.: On the eigenstates of the elliptic Calogero-Moser model, Lett. Math. Phys. 53, 181 (2000)

[WW] Whitaker E. T. and Watson G. N.: Course of modern analysis, 4th edition. Cambridge Univ. Press (1958) 